\title{
Efficient thermal spin injection using CoFeAl nanowire
}

\author{
Shaojie $\mathrm{Hu}^{1}$, Hiroyoshi Itoh ${ }^{2,3}$ and Takashi Kimura ${ }^{3,4}$ \\ Nanoelectronic devices based on electron spin can overcome the physical limitations of the present semiconductor technology \\ because of their low power consumption while exploiting the spin degree of freedom of electrons. Although enhancing the \\ efficiency of generation of the spin current is imperative and a primary issue for the practical application of spin-based \\ electronics, seamless device integration with the conventional complementary metal-oxide semiconductor technology is another \\ important milestone for developing spin-based nanoelectronics. In particular, the preparation of nanosized, magnetic, \\ multilayered structures with electrical connections to individual complementary metal-oxide semiconductor circuits significantly \\ complicates the fabrication procedure of nanoelectronic devices. Thermal spin injection, which is a recently discovered unique \\ characteristic of spin current, may be an innovative method for simplifying device integration without the need for electricity, \\ namely wireless spintronics. However, the feasibility of using the thermal spin injection method is poor because of its extremely \\ low-generation efficiency. Here, we demonstrate that a highly spin-polarized, ferromagnetic CoFeAl electrode with a favorable \\ band structure has excellent properties for thermal spin injection. The spin-dependent Seebeck coefficient is approximately \\ $70 \mu \mathrm{VK}^{-1}$, which facilitates highly efficient generation of the spin current from heat. The heat generates approximately 100 \\ times more spin voltage than a conventional ferromagnetic injector at room temperature. This innovative demonstration may \\ open a new route for spin-device integration and its applications.
}

NPG Asia Materials (2014) 6, e127; doi:10.1038/am.2014.74; published online 19 September 2014

\section{INTRODUCTION}

Spin current is central to the operation of spin-based nanoelectronic devices. ${ }^{1-5}$ The efficient manipulation of spin current is essential for developing functional and energy-efficient nanospintronic devices. Recently, heat has been used as a new approach to control the spin in ferromagnetic/nonmagnetic hybrid nanostructures. ${ }^{6-14}$ A representative and fascinating phenomenon is thermal spin injection, in which excess heat can be used to produce spin current because of the spin-dependent Seebeck coefficient. ${ }^{7-9}$ Until now, thermally driven spin injection has only been demonstrated using conventional ferromagnetic metals such as permalloy $(\mathrm{Py})^{7}$ and cobalt. ${ }^{13}$ However, thermally excited spin current can only generate a few tens of $\mathrm{nV}$ which is low, because of the low spin-dependent Seebeck coefficient of conventional ferromagnetic metals.

In electrical spin injection, the difference in the electrical conductivity between the up and down spins produces spin current. ${ }^{4,5}$ However, because the direction of movement of the electrons is determined by the electric field, the electrons with up spins move in the same direction as those with down spins. Therefore, the generation efficiency of the spin current cannot exceed $100 \%$ even when a fully spin-polarized material, that is, a half-metal, is used. Furthermore, the spin current induced by thermal spin injection is determined by the difference in the Seebeck coefficient between the up and down spins, ${ }^{7,15}$ which is known as the spin-dependent Seebeck effect. This effect is different from the spin Seebeck effect, ${ }^{6,14}$ although both effects produce spin current and spin accumulation from the temperature gradient. The Seebeck coefficient is strongly correlated to the band structure around the Fermi level, and under simple approximation in metals, the coefficient is proportional to the energy derivative of the logarithmic density of state (DOS) at the Fermi level. ${ }^{16-18}$ In a ferromagnetic material, because the DOS has different features for up and down spins, one must separately consider the movement directions of the up-spin electrons and the down-spin electrons.

For conventional ferromagnets, the difference in the band structure between the up and down spins can be approximated as a small shift in the DOS. In this case, although the up-spin and down-spin electrons produce a Seebeck effect of a different magnitude, the difference is small. Moreover, electrons of either spin diffuse in the same direction, as shown in Figures $1 \mathrm{a}$ and b. Therefore, the magnitude of the spin current diminishes. If a ferromagnetic material has a large difference in the DOS between up and down spins, a sign reversal of the Seebeck coefficient between the up and down spins may be produced. In this situation, the up-spin and down-spin electrons flow in opposite directions, as shown in Figures 1c and d. Therefore, the generation efficiency of the spin current that occurs because of thermal spin injection is significantly enhanced by the large spin-dependent Seebeck coefficient.

To realize this favorable situation for efficient thermal spin injection, we focused on a ferromagnetic CoFeAl (CFA) alloy, which

${ }^{1}$ Graduate School of Information Science and Electrical Engineering, Kyushu University, Fukuoka, Japan; ${ }^{2}$ Department of Pure and Applied Physics, Kansai University, Suita, Japan; ${ }^{3}$ CREST, Japan Science and Technology Agency, Tokyo, Japan and ${ }^{4}$ Department of Physics, Kyushu University, Fukuoka, Japan

Correspondence: Professor T Kimura, Department of Physics, Kyushu University, 6-10-1 Hakozaki, Fukuoka 812-8581, Japan.

E-mail: t-kimu@phys.kyushu-u.ac.jp

Received 28 February 2014; revised 20 July 2014; accepted 22 July 2014 
a

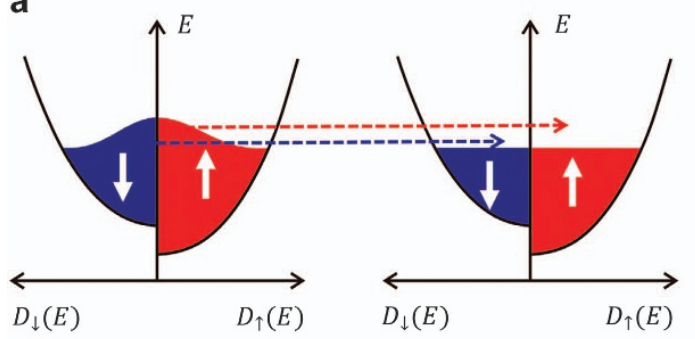

C

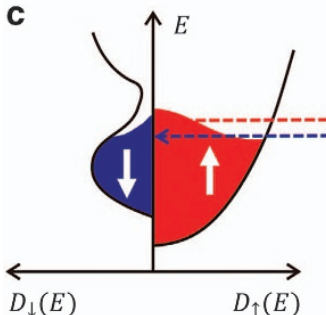

b
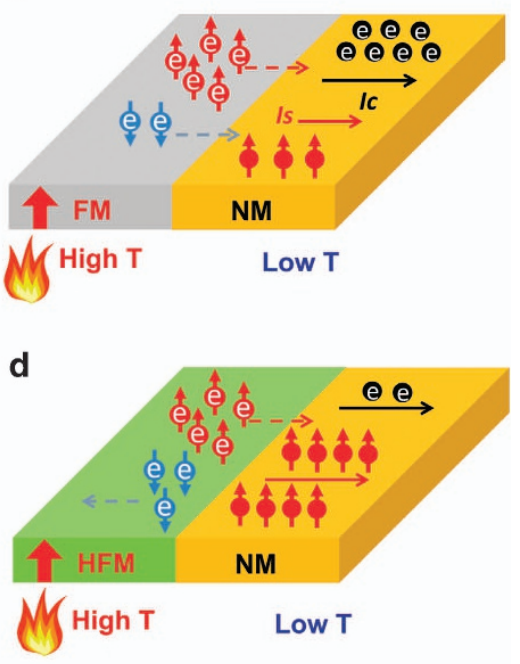

Figure 1 Conceptual illustrations of highly efficient thermal spin injection. (a) Schematic illustration of the spin-dependent density of states (DOSs) for a conventional ferromagnetic metal at high (left) and low (right) temperatures. Both the up-spin and down-spin electrons diffuse from the left-hand side (high temperature) to the right-hand side (low temperature). (b) Charge and spin current flow in the conventional ferromagnet/nonmagnet junction under the temperature gradient. The generation and injection efficiencies are low because both up-spin and down-spin electrons move in the same direction. (c) Schematic illustration of the spin-dependent DOSs of a highly spin-polarized ferromagnetic metal at high (left) and low (right) temperatures. The small DOS of the minority spin around the Fermi level reverses the movement direction of the electrons under the temperature gradient. As a result, the down-spin electrons move in the opposite direction from the up-spin electrons. (d) Charge and spin current flow in the highly spin-polarized ferromagnet/ nonmagnet junction under the temperature gradient. The spin current is efficiently generated and injected into the nonmagnet.

is expected to have high spin polarization even in a simply evaporated polycrystalline film because of the local formation of a highly spinpolarized Heusler compound. ${ }^{19,20}$ This characteristic is an important advantage for preparing multiterminal functional lateral spin valve (LSV) structures because no specific substrate or thermal treatment is required during the sample fabrication process.

\section{EXPERIMENTAL PROCEDURE}

Our CFA film was deposited using electron-beam evaporation from a CFA melting source, whose composition was $48 \mathrm{wt} \% \mathrm{Co}-48 \mathrm{wt} \% \mathrm{Fe}-4 \mathrm{wt} \%$ Al. The composition of the evaporated film and its uniformity were confirmed using an energy dispersive X-ray detector in a scanning electron microscope system. The obtained composition of the evaporated film was $43 \mathrm{wt} \%$ $\mathrm{Co}-54 \mathrm{wt} \% \mathrm{Fe}-3 \mathrm{wt} \% \mathrm{Al}$ from the energy dispersive X-ray analysis. It should be noted that the $\mathrm{Al}$ content was considerably different from the previously reported values. ${ }^{19,20}$

A batch of conventional LSV structures with two CFA wires bridged by a nonmagnetic $\mathrm{Cu}$ wire was fabricated on a $\mathrm{SiO}_{2} / \mathrm{Si}$ substrate using electron beam lithography and the lift-off technique. Figure 2a shows a scanning electron microscope image of a representative device in our study. First, two $\mathrm{CoFeAl}$ wires, each of which was $30 \mathrm{~nm}$ thick and $120 \mathrm{~nm}$ wide, were prepared using an electron beam evaporator with a base pressure of approximately $10^{-9}$ torr. We introduced a difference in the edge shapes between the two wires to easily control the magnetization configuration using an external magnetic field. The interval distances $(L)$, which correspond to the center-center distance between the two wires, were varied from 200 to $1500 \mathrm{~nm}$ on the same chip. Then, the $\mathrm{Cu}$ channel wires, each of which was $160 \mathrm{~nm}$ thick and $120 \mathrm{~nm}$ wide, were deposited using Joule heating evaporation. The surfaces of the CoFeAl wires were carefully cleaned using low-voltage Ar ion milling before depositing $\mathrm{Cu}$ to obtain a highly transparent interface (see Supplementary Information). The resistivity of the CFA wires was evaluated from the four terminal resistance of a well-defined wire in the same batch and estimated to be $4.5 \pm 0.2 \times 10^{-7} \Omega \mathrm{m}$ at room temperature, which is one order of magnitude smaller than the previously reported value. Because the present value is close to the value for CoFe alloy, ${ }^{20}$ the difference in resistivity is most likely because of the difference in $\mathrm{Al}$ content. The electrically and thermally driven pure spin currents were detected using a low-frequency lock-in technique with the first- and second-harmonic voltage responses, respectively.

\section{RESULTS AND DISCUSSION}

First, we evaluated the electrical spin polarization of the CFA wires by measuring the nonlocal spin signal under the electrical spin injection, as shown in Figure 2a. The electric current across the ferromagnetic/ nonmagnetic interface creates spin accumulation in the nonmagnetic $\mathrm{Cu}$ channel. This nonequilibrium spin accumulation produces a spin current without an accompanying charge current, that is, a pure spin current, on the upside of the nonmagnetic channel., ${ }^{5,21,22}$ The pure spin current can be detected using another ferromagnetic electrode after it propagates in the nonmagnetic channel. As shown in Figure $2 \mathrm{~b}$, the nonlocal electrical voltage shows a clear spin valve signature with an overall resistance change of more than $10 \mathrm{~m} \Omega$ at room temperature. For comparison, the spin signal for a similarly fabricated Py/Cu LSV with the same device dimensions is shown in Figure $2 \mathrm{~b}$. We confirmed that the spin signal for the CFA/Cu LSV was 10 times larger than that for the Py/Cu LSV, which indicates the superior properties of our developed CFA injector.

To elucidate the main mechanism of the significant enhancement of spin signal in the CFA/Cu LSV, we measured the interval dependence of the spin signals for both $\mathrm{CFA} / \mathrm{Cu}$ and $\mathrm{Py} / \mathrm{Cu}$ LSVs. As shown in Figure 2c, the dependences of the spin signal on the interval $L$ for both devices were well fitted using the one-dimensional spin diffusion equation with transparent interfaces, which is given by ${ }^{23-25}$

$$
\Delta R_{s}^{1 f}=\frac{P_{F}^{2} R_{F}^{2} R_{N}}{2 R_{F}\left(R_{F}+R_{N}\right)\left(\cosh \left(L / \lambda_{N}\right)+\sinh \left(L / \lambda_{N}\right)\right)+R_{N}^{2} \sinh \left(L / \lambda_{N}\right)}
$$

where $P_{\mathrm{F}}$ is the spin polarization of the ferromagnet, $\lambda_{\mathrm{N}}$ is the spin diffusion length of the nonmagnet and $R_{\mathrm{F}}$ and $R_{\mathrm{N}}$ are the spin resistances of the ferromagnet and nonmagnet, respectively. 

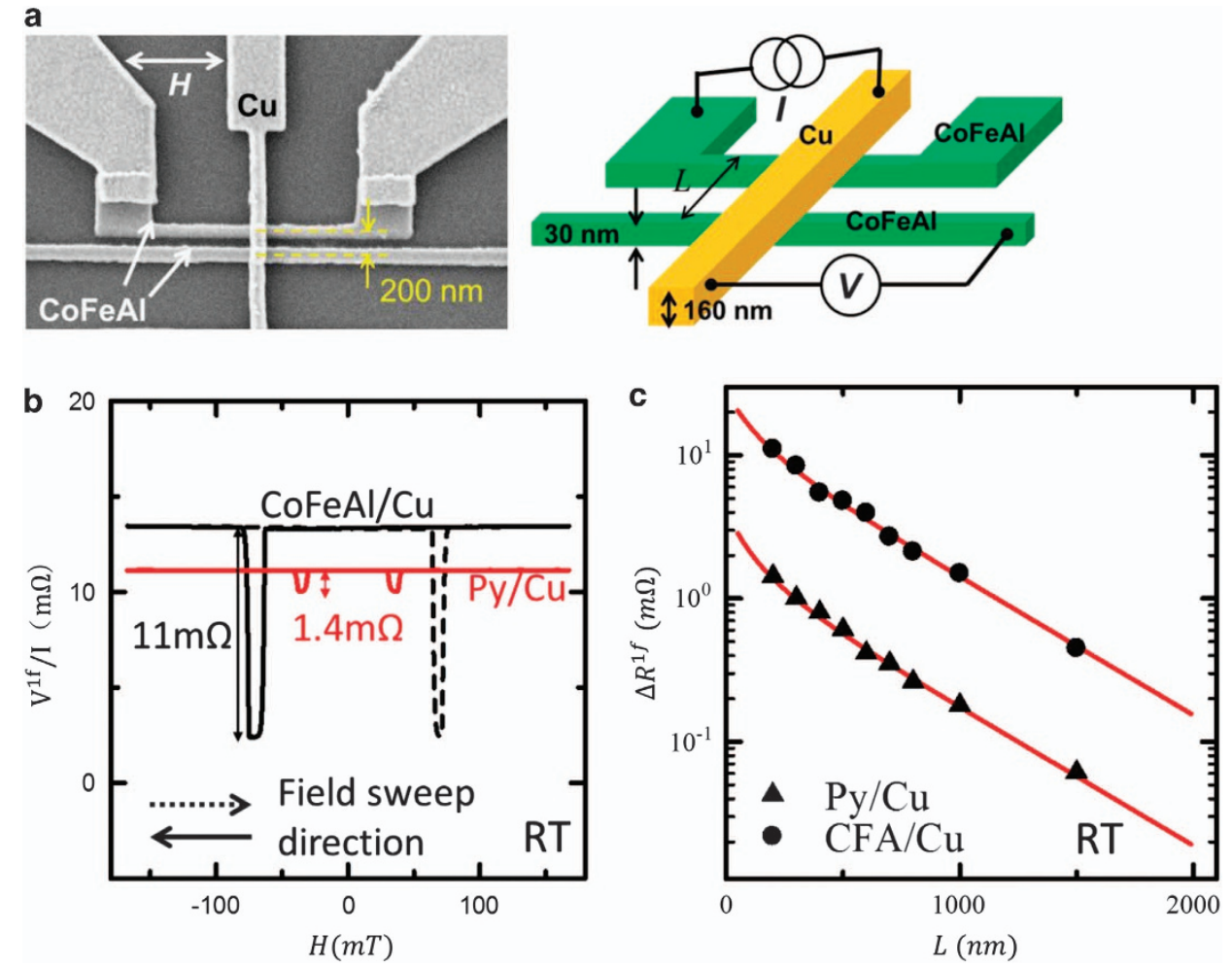

Figure 2 Evaluation of electrical spin polarization. (a) Scanning electron microscope image of the CoFeAl (CFA)/Cu lateral spin valve (LSV) in the present study and a schematic figure of the nonlocal spin valve measurement to evaluate the electrical spin polarization. (b) Room temperature nonlocal spin signals for the CoFeAl/Cu (black curve) and permalloy (Py)/Cu (red curve) LSVs at the center-center distance $L$ of $200 \mathrm{~nm}$. The dotted and solid curves denote the forward and backward field sweeps, respectively. (c) Room temperature spin signal as a function of the interval distance $(L)$ for CoFeAl/Cu (solid dots) and Py/Cu (solid triangles) LSVs. The red lines are the fitted curves based on Equation (1) with $\lambda_{\mathrm{Cu}}=450 \mathrm{~nm}$ at room temperature.

The spin resistance $R_{\mathrm{s}}$ is defined as $2 \rho \lambda /\left(A\left(1-P^{2}\right)\right)$, where $P, \rho$ and $\lambda$ are the spin polarization, electrical resistivity and spin diffusion length, respectively. For a nonmagnet, $P$ should be zero. $A$ is the effective cross-section of the spin current. For the ferromagnet, because the spin diffusion length is short, the effective cross-section is given by the area of the ferromagnetic/nonmagnetic junction.

It should be noted that the estimated spin diffusion length of $\mathrm{Cu}$ from the fittings for both CFA/Cu and Py/Cu LSVs was $450 \mathrm{~nm}$. If we assume that CFA and Py have identical spin diffusion lengths of $2 \mathrm{~nm},{ }^{25}$ the spin polarizations of CFA and Py can be estimated as 0.62 and 0.36 , respectively. Thus, the strong enhancement of the spin signal by a factor of 10 is attributed to the efficient generation and detection of the spin current because CFA has a large spin polarization and the spin current backflow is significantly reduced. ${ }^{26}$ The obtained spin polarization of the present CFA is close to the previously reported values, although the $\mathrm{Al}$ content and electrical resistivity are notably different, ${ }^{19,20}$ which suggests that our CFA has a different crystal structure from those in the previous reports.

Then, we performed thermal spin injection by changing the probe configuration in the same device. To create a temperature gradient across the ferromagnetic/nonmagnetic interface, which is the driving force of thermal spin injection, a large AC charge current flowed in the ferromagnetic wire, as shown in Figure 3a. From a numerical simulation based on COMSOL, a large temperature gradient around the interface was effectively produced by the large current flow in the ferromagnetic injector, as shown in Figure $3 b .^{7,27}$ Because of the low resistivity of $\mathrm{Cu}$, a partial current flowing in the ferromagnetic injector was injected into the $\mathrm{Cu}$ around the junction, which might contribute to the spin accumulation using electrical spin injection. However, such contributions were excluded using the secondharmonic lock-in technique. In addition, the contribution of the electrical spin injection was confirmed to be negligible by the first-harmonic measurement (see Supplementary Information).

We measured the second-harmonic voltage under the large AC current flow as a function of the magnetic field in the CFA/Cu LSV. As seen in Figure $3 c$, the voltage clearly reflected the relative angle between the spin injector and the detector, which was similar to the spin signal under the electrical spin injection. We performed similar measurements using the Py/Cu LSV with identical device dimensions. The signal was dominated by the anomalous Nernst-Ettinghausen effect in the Py detector, although a tiny spin signal with a magnitude of $7 \mathrm{nV}$ was observed. ${ }^{28}$

We also confirmed that the voltage difference between the parallel and antiparallel states parabolically increased with the increase in AC bias current, as shown in Figure 3d. In addition, the interval dependence of the voltage difference is well explained using the following equation, which was obtained from the one-dimensional spin diffusion model (see Supplementary Information):

$$
\Delta V_{s}^{2 f}=\frac{P_{F} R_{N} R_{F} \lambda_{F} \nabla T_{F} S_{s}}{2 R_{F}\left(R_{F}+R_{N}\right)\left(\cosh \left(L / \lambda_{N}\right)+\sinh \left(L / \lambda_{N}\right)\right)+R_{N}^{2} \sinh \left(L / \lambda_{N}\right)}
$$

where $\nabla T_{\mathrm{F}}$ is the temperature gradient in the ferromagnet around the interface and $S_{\mathrm{s}}$ is the spin-dependent Seebeck coefficient of the ferromagnetic injector. The parameters obtained in the electrical spin injection experiments are consistent with the thermal spin injection experimental results. These characteristics are clear evidence of 


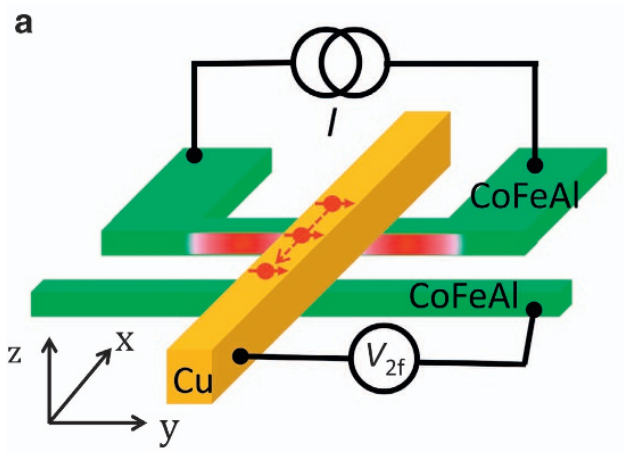

C

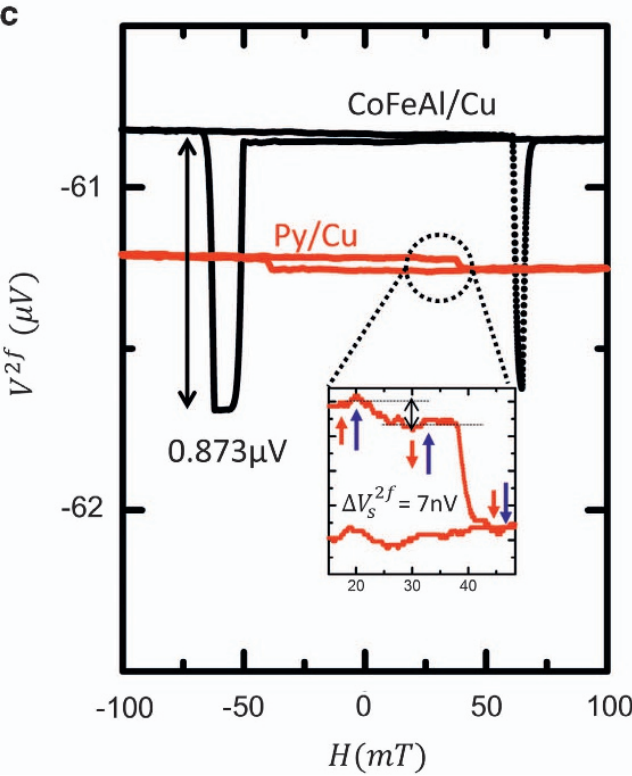

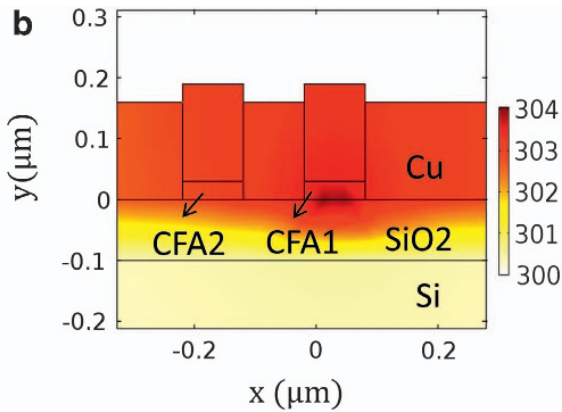

d
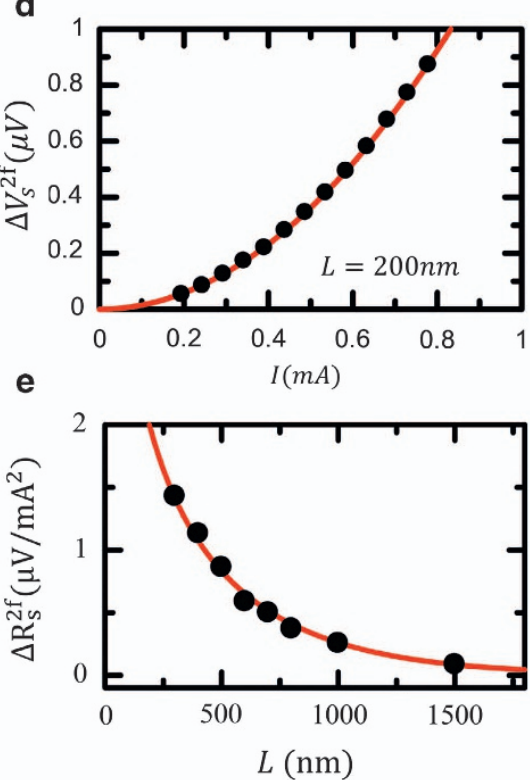

Figure 3 Giant spin accumulation because of thermal spin injection. (a) Schematic illustration of thermal spin injection and detection using the lateral spin valve (LSV) structure. A thermally excited spin current is generated by flowing a large current in a ferromagnetic wire (F1), which is detected using a ferromagnetic detector (F2) with the second-harmonic voltage detection technique. (b) Numerically simulated result of the spatial distribution of temperature around the CoFeAl (CFA)/nonmagnetic (NM) injecting junction under a large current of $0.78 \mathrm{~mA}$. Here, the current value corresponds to the root mean square. The Joule heating induced by the current flow in the ferromagnetic wire clearly produces a temperature gradient across the ferromagnetic (FM)/NM interface. (c) Field dependence of the second-harmonic voltage under thermal spin injection for CoFeAl/Cu LSV (black line) with a 200 nm interval distance under a bias current of $0.78 \mathrm{~mA}$ and the second-harmonic signal of the Py/Cu LSV with the same sample dimensions under a bias current of $1.08 \mathrm{~mA}$. The asymmetric field dependence is induced by the anomalous Nernst effect in the Py detector. (d) Bias current dependence of the secondharmonic spin voltage $\Delta V^{2 f}$ for the CFA/Cu LSV. The red line is the fitting curve with the assumption of the parabolic dependence. (e) The thermal spin signal, which is defined as $\Delta \mathrm{R}_{\mathrm{s}}^{2 f}=\Delta \mathrm{V}_{\mathrm{s}}^{2} / \mathrm{I}^{2}$, as a function of the interval distance $L$ for the CFA/Cu LSV at room temperature. The solid red line is a fitted curve based on Equation (2), assuming $\lambda_{\mathrm{Cu}}=450 \mathrm{~nm}$.

thermal spin injection. As shown in Figure 3e, by fitting the diffusion function to the interval dependence of the thermal spin signal, which is defined as $\Delta \mathrm{R}_{\mathrm{s}}^{2 \mathrm{f}}=\Delta \mathrm{V}_{\mathrm{s}}^{2} / I^{2}$, with the COMSOL simulation results

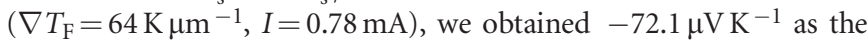
spin-dependent Seebeck coefficient for the CFA (see Supplementary Information). This value is $\sim 20$ times larger than the previously reported value in Slachter et al., ${ }^{7}$ which implies that our CFA is a highly efficient thermal spin generator.

We can also estimate the effective Seebeck coefficient $S_{0}$ from the second-harmonic voltage background. ${ }^{29}$ The background voltage parabolically increased with increasing bias AC current. From the fitting with the assumption, we obtained a conventional Seebeck coefficient of $-22 \mu \mathrm{VK}^{-1}$. Notably, the spin-dependent Seebeck coefficient was larger than the conventional Seebeck coefficient. From the relationships of $S_{0}=\frac{S_{\uparrow} \sigma_{\uparrow}+S_{\downarrow} \sigma_{\downarrow}}{\sigma_{\uparrow}+\sigma_{\downarrow}}$ and $S_{\mathrm{s}}=S_{\uparrow}-S_{\downarrow}$, the calculated Seebeck coefficients for up-spin $\left(S_{\uparrow}\right)$ and down-spin $\left(S_{\downarrow}\right)$ electrons were -35.7 and $36.4 \mu \mathrm{VK}^{-1}$, respectively. Thus, the spin-dependent Seebeck coefficient was significantly enhanced because of the sign reversal of the Seebeck coefficient. We note that some of the band-structure calculations show that the energy derivatives for the DOSs around the Fermi level has opposite signs for up and down spins. ${ }^{30,31}$ Understanding the correlation between the electrical spin polarization and the spin-dependent Seebeck coefficient may be important for further development of thermal spin injection and may provide a relationship between the spindependent Seebeck effect and the spin Seebeck effect. ${ }^{32}$ Systematic experiments for various ferromagnetic alloys with further development of the band-structure calculations will be indispensable to find more excellent materials for the thermal spin injector.

\section{CONCLUSION}

The temperature gradient that is the driving force of thermal spin injection can be produced using direct physical contact with a hot object, laser irradiation or microwave absorption. Efficient spin 
current generation using thermal spin injection provides unique architectures for wireless spintronic devices, which greatly simplifies device integration. ${ }^{33}$ In addition, from the energy-harvesting viewpoint, heat usage is one of the most promising approaches for clean-energy technology. The discovery of excellent materials for thermal spin injection opens a new path for spintronic-device application.

1 Wolf, S. A., Awschalom, D. D., Buhrman, R. A., Daughton, J. M., von Molnár, S., Roukes, M. L., Chtchelkanova, A. Y. \& Treger, D. M. Spintronics: a spin-based electronics vision for the future. Science 294, 1488-1495 (2001).

2 Chappert, C., Fert, A. \& Van Dau, F. N. The emergence of spin electronics in data storage. Nat. Mater. 6, 813-823 (2007).

3 Zutic, I. \& Das Sarma, S. Spintronics: fundamentals and applications. Rev. Mod. Phys. 76, 323-410 (2004)

4 Maekawa, S., Valenzuela, S. O., Saitoh, E. \& Kimura, T. Spin Current (Oxford University Press, Oxford, UK, 2012)

5 Bakaul, S., Hu, S. \& Kimura, T. Large pure spin current generation in metallic nanostructures. Appl. Phys. A 111, 355-360 (2013).

6 Bauer, G. E. W., Saitoh, E. \& van Wees, B. J. Spin caloritronics. Nat. Mater. 11, 391-399 (2012).

7 Slachter, A., Bakker, F. L., Adam, J.-P. \& van Wees, B. J. Thermally driven spin injection from a ferromagnet into a non-magnetic metal. Nat. Phys. 6, 879-882 (2010).

8 Le Breton, J.-C., Sharma, S., Saito, H., Yuasa, S. \& Jansen, R. Thermal spin current from a ferromagnet to silicon by Seebeck spin tunnelling. Nature 475, 82-85 (2011).

9 Walter, M., Walowski, J., Zbarsky, V., Münzenberg, M., Schäfers, M., Ebke, D., Reiss, G.. Thomas, A., Peretzki, P., Seibt, M., Moodera, J. S., Czerner, M., Bachmann, M. \& Heiliger, C. Seebeck effect in magnetic tunnel junctions. Nat. Mater. 10, 742-746 (2011)

10 Erekhinsky, M., Casanova, F., Schuller, I. K. \& Sharoni, A. Spin-dependent Seebeck effect in non-local spin valve devices. Appl. Phys. Lett. 100, 212401 (2012).

11 Bakaul, S. R., Hu, S. \& Kimura, T. Thermal gradient driven enhancement of pure spin current at room temperature in nonlocal spin transport devices. Phys. Rev. B 88, 184407 (2013)

12 Johnson, M. \& Silsbee, R. Thermodynamic analysis of interfacial transport and of the thermomagnetoelectric system. Phys. Rev. B 35, 4959-4972 (1987).

13 Gravier, L., Serrano-Guisan, S., Reuse, F. \& Ansermet, J. P. Thermodynamic description of heat and spin transport in magnetic nanostructures. Phys. Rev. B 73, 024419 (2006).

14 Uchida, K., Takahashi, S., Harii, K., leda, J., Koshibae, W., Ando, K., Maekawa, S. \& Saitoh, E. Observation of the spin Seebeck effect. Nature 455, 778-781 (2008).

15 Scharf, B., Matos-Abiague, A., Zutic, I. \& Fabian, J. Theory of thermal spin-charge coupling in electronic systems. Phys. Rev. B 85, 085208 (2012).

16 Mott, N. F. \& Davis, E. A. Electronic Processes in Non-Crystalline Materials 52 (Clarendon Press, Oxford, UK, 1979).

17 Heremans, J. P. Low-dimensional thermoelectricity. Acta Phys. Pol. A 108, 609-634 (2005).
18 Pichard, C. R., Tellier, C. R. \& Tosser, A. J. Thermoelectric power of thin polycrystalline metal films in an effective mean free path model. J. Phys. F 10, 2009-2014 (1980).

19 Wurmehl, S., Jacobs, P. J., Kohlhepp, J. T., Swagten, H. J. M., Koopmans, B., Maat, S. Carey, M. J. \& Childress, J. R. Local formation of a Heusler structure in CoFe-Al alloys. Appl. Phys. Lett. 98, 012506 (2011).

20 Bridoux, G., Costache, M. V., Van de Vondel, J., Neumann, I. \& Valenzuela, S. O. Enhanced spin signal in nonlocal devices based on a ferromagnetic CoFeAl alloy. Appl. Phys. Lett. 99, 102107 (2011).

21 Johnson, M. \& Silsbee, R. H. Interfacial charge-spin coupling: injection and detection of spin magnetization in metals. Phys. Rev. Lett. 55, 1790-1793 (1985).

22 Jedema, F. J., Filip, A. T. \& van Wees, B. J. Electrical spin injection and accumulation at room temperature in an all-metal mesoscopic spin valve. Nature 410, 345-348 (2001).

23 Valet, T. \& Fert, A. Theory of the perpendicular magnetoresistance in magnetic multilayers. Phys. Rev. B 48, 7099-7113 (1993).

24 Takahashi, S. \& Maekawa, S. Spin injection and detection in magnetic nanostructures. Phys. Rev, B 67, 052409 (2003).

25 Kimura, T., Hamrle, J. \& Otani, Y. Estimation of spin-diffusion length from the magnitude of spin-current absorption: multiterminal ferromagnetic/nonferromagnetic hybrid structures. Phys. Rev. B 72, 1-6 (2005).

26 Kimura, T., Hashimoto, N., Yamada, S., Miyao, M. \& Hamaya, K. Room-temperature generation of giant pure spin currents using epitaxial $\mathrm{Co}_{2} \mathrm{FeSi}$ spin injectors. NPG Asia Mater. 4, e13 (2012).

27 Slachter, A., Bakker, F. L. \& van Wees, B. J. Modeling of thermal spin transport and spin-orbit effects in ferromagnetic/nonmagnetic mesoscopic devices. Phys. Rev. B 84, 174408 (2011).

$28 \mathrm{Hu}, \mathrm{S}$. \& Kimura, T. Anomalous Nernst-Ettingshausen effect in nonlocal spin valve measurement under high-bias current injection. Phys. Rev. B 87, 014424 (2013).

29 Bakker, F. L., Slachter, A., Adam, J. P. \& van Wees, B. J. Interplay of Peltier and Seebeck effects in nanoscale nonlocal spin valves. Phys. Rev. Lett. 105, 136601 (2010).

30 Katsnelson, M., Irkhin, V., Chioncel, L., Lichtenstein, A. \& de Groot, R. Half-metallic ferromagnets: from band structure to many-body effects. Rev. Mod. Phys. 80, 315 (2008).

31 Comtesse, D., Geisler, B., Entel, P., Kratzer, P. \& Szunyogh, L. Electronic, magnetic and transport properties of full and half-metallic thin film Heusler alloys. Arxiv 1331, 1950 (2013).

32 Bosu, S., Sakuraba, Y., Uchida, K., Saito, K., Ota, T., Saitoh, E. \& Takanashi, K. Spin Seebeck effect in thin films of the Heusler compound $\mathrm{C}_{2}$ MnSi. Phys. Rev. B 83, 1 (2011).

33 Behin-Aein, B., Datta, D., Salahuddin, S. \& Datta, S. Proposal for an all-spin logic device with built-in memory. Nat. Nanotechnol. 5, 266-270 (2010).

This work is licensed under a Creative Commons Attribution 3.0 Unported License. The images or other third party material in this article are included in the article's Creative Commons license, unless indicated otherwise in the credit line; if the material is not included under the Creative Commons license, users will need to obtain permission from the license holder to reproduce the material. To view a copy of this license, visit http:// creativecommons.org/licenses/by/3.0/

Supplementary Information accompanies the paper on the NPG Asia Materials website (http://www.nature.com/am) 